\title{
SIMULATIONS OF THE GRAVITATIONAL MICROLENSING: EXTENDED SOURCE MODELS AND IMPACT OF BINARY STARS
}

\author{
V. M. Sliusar, V. I. Zhdanov, A. N. Alexandrov \\ Astronomical Observatory, Taras Shevchenko National University of Kyiv, \\ 3, Observatorna St., UA-04053, Kyiv, Ukraine
}

(Received January 12, 2012; received in final form October 19, 2012)

\begin{abstract}
In the extragalactic gravitational lens system several images of a distant quasar are observed through a foreground galaxy. The observation of light curves of different quasar images provides an important information about the central quasar region. We perform statistical simulations of the light curves of the microlensed images to estimate a feasibility to distinguish source models with different brightness profiles. Two cases of mass distribution in the foreground lensing galaxy are studied: (i) random distribution of stars represented by single point masses and (ii) analogous distribution of double stars. We found that the difference between light curves for the accretion disk and Gaussian source model of the same radius can reach up to $8 \%$ in some cases. If we fit the accretion disk and the limb darkening source models with the Gaussian surface brightness profile and the size is allowed to be not the same, then that this difference can be decreased to $7 \%$ and $2.6 \%$, respectively. A comparison of the results shows that the difference between cases (i) and (ii) is at the limit of statistical relevance, though some light curves may differ considerably.
\end{abstract}

Key words: gravitational lensing, extragalactic microlensing, accretion disk models.

PACS number(s): 98.62.Sb

\section{INTRODUCTION}

In a gravitational lens system (GLS) there is a gravitating object (galaxy, cluster, isolated star, etc.) near the line of sight to a distant source. The gravitational field of this object ("gravitator") acts as an optical lens: it creates several source images, distorts these images and amplifies their brightness. Observation of the source image distortions in GLS and the image light curves yields a unique information concerning the gravitator and about the source structure.

In this paper we pay a main attention to extragalactic GLSs which typically have several macro-images of one quasar. The light from the quasar corresponding to different images passes through the lensing galaxy in different regions. The gravitational field of the lensing galaxy in these regions, which is formed by gravitational fields of separate stars, is highly inhomogeneous; it creates a huge caustic network in the source plane. Because of this inhomogeneity every macro-image is a collection of a number of micro-images that cannot be resolved in observations. The relative motion of the source and the gravitator leads to considerable changes of brightness of every macro-image; typical timescales of these changes vary from days to months. Local gravitational fields of the lensing galaxy on the lines of sight of the different macro-images are practically independent. Therefore the microlensing brightness variations in different macroimages are also independent; this enables one to distinguish the microlensing effects in observations from the proper brightness variations of the quasar itself.

Sometimes a considerable brightness enhancement in some micro-image occurs which is referred to as a high amplification event (HAE); it is associated with an in- tersection of the GLS caustic by the source. One of important applications of HAE deals with a possibility to study a light distribution over the source in GLS. This is especially interesting for extragalactic systems in connection with investigation of the central region of distant quasars. The idea, first proposed by Grieger, Kayser and Refsdal [1], appeals to an approximate formula of flux amplification during HAE, which contains a few fitting parameters arising in the linear caustic approximation of the lens equation. This makes possible estimations of certain GLS characteristics, in particular, the source size [1]. For example, in case of well known GLS Q2237 +0305 (Einstein Cross) several HAEs was observed [2-4] and the estimates of the source size have been obtained within different source models [5-11], see also [5-7, 12-17].

Under the assumption that some HAE is attributed just to the fold caustic crossing in the source plane, in case of a sufficient photometric accuracy it is possible to estimate additional parameters that arise in the postlinear approximations of the lens equation $[18,19]$. For example, taking into account additional parameters in the expansion of the lens equation near the fold allows one to reduce $\chi^{2}$ by $30 \%$ to obtain an optimal value in case of fitting [18] the HAE light curve of one of the images of Q2237+030 GLS (Einstein Cross). The possibility do distinguish different source models is also discussed elsewhere (see, e. g., references in [20]).

The aim of this paper is twofold. First, we compare the results of simulations with different source models. Second, we estimate a difference of statistical characteristics in case of different mass distributions in the foreground lensing galaxy: (i) random distribution of single stars represented by single equal point masses and (ii) analogous distribution of binary stars having in mind that much 
of the stars are in binary systems. In Section II we outline the statement of the problem: the lens equation and the source models used (Gaussian, power-law and accretion disc models). Section III contains the parameters of simulations and statistical results of a comparison of different source models in cases (i) and (ii). In the Discussion we outline the main problems arising in such a comparison.

\section{GENERAL RELATIONS AND NOTATIONS}

\section{A. The lens equation}

We assume zero external shear and zero optical depth of the continuous matter. In case of equal microlensing masses the lens equation is as follows:

$$
\mathbf{y}=\mathbf{x}-R_{\mathrm{E}}^{2} \sum_{i=1}^{N} \frac{\mathbf{x}-\mathbf{x}_{\mathbf{i}}}{\left|\mathbf{x}-\mathbf{x}_{\mathbf{i}}\right|^{2}}
$$

where $\mathbf{x}_{\mathbf{i}}$ is the angular position of the $i$-th microlens on the sky, $R_{\mathrm{E}}$ is its angular Einstein ring radius:

$$
R_{\mathrm{E}}=\sqrt{\frac{4 G M}{c^{2}} \frac{D_{\mathrm{ds}}}{D_{\mathrm{d}} D_{\mathrm{s}}}}
$$

where $D_{\mathrm{s}}$ stands for the observer-source distance, $D_{\mathrm{d}}$ is observer-deflector distance, $D_{\mathrm{ds}}$ is the deflector-source distance (here we deal with the angular diameter distances).

In the numerical calculations the "ray-shooting" method is used, it was initially proposed in [21]. This method defines an amplification of a source with any brightness profile $[22,23]$; it is very effective and fast.

The enhancement of the basic method is related with the hierarchical tree-code (see, e.g., [22-24]); though in our simulations this was not used.

\section{B. Extended source models}

One of the main characteristics that have an impact on the amplification of the microlensed source is its brightness profile - the distribution of brightness over the source disk. Quasars that are microlensed by stars of the deflecting galaxy have still directly unresolvable surface brightness distributions. So it is interesting to study whether we can distinguish different source models using microlensing observations on account of modern accuracy of photometric observations.

To compare models with diverse brightness distribution one must use the same parameter that characterizes the size of a source. The most general is the r.m.s. radius. However, for slowly decreasing brightness profile (e.g., if for large $|\mathbf{y}|$ we have $I(\mathbf{y}) \sim|\mathbf{y}|^{-\alpha}, \quad \alpha \leq 4$ ) the r. m. s. size diverges. The other characteristic can be proposed in terms of the coherence area [25] (see Appendix
1). Though this characteristic is less used in microlensing considerations, it is free from the latter divergence for large $|\mathbf{y}|$. Although, in this case we may have another divergence, if $I(\mathbf{y}) \sim|\mathbf{y}|^{-\beta}, \beta \geq 2$ for $|\mathbf{y}| \rightarrow 0$. Anyway, in our consideration the choice of a characteristic size parameter is not essential. Indeed, in the comparison of different models one must fit them to observational data independently yielding different values of size parameters. This is just the way we follow in Section III C. Nevertheless, in the previous Section we compare preliminarily the models with the same size parameter. As we see, this is also quite reasonable because fitting with different parameters in Section III C yields almost the same results.

In this connection we prefer to use the half-brightness radius $R_{1 / 2}$; it is defined in case of circular symmetric brightness distributions by the relation:

$$
\int_{0}^{R_{1 / 2}} I(r) r d r=\frac{1}{2} \int_{0}^{\infty} I(r) r d r .
$$

The simplest model used for the calculations of light curves is the Gaussian one:

$$
I_{\mathrm{G}}(r)=\frac{1}{\pi R^{2}} \exp \left[-\left(\frac{r}{R}\right)^{2}\right]
$$

where $R_{1 / 2}=R \sqrt{\ln (2)}, R$ stands for a size parameter.

The limb-darkening (LD) model with the power in$\operatorname{dex} q$ (see, e.g., [26]) is mostly appropriate to describe a stellar surface:

$$
I_{\mathrm{LD}}(r)=\frac{q+1}{\pi R^{2}} \Xi(r / R ; q)
$$

where we denoted $\Xi(\xi ; q)=\Theta\left(1-\xi^{2}\right)\left(1-\xi^{2}\right)^{q}, \quad q \geq 0$, and $\Theta(x)$ is the Heaviside step function.

According to (3) for the LD model $R_{1 / 2}=$ $R \sqrt{1-(1 / 2)^{1 /(q+1)}}$. For the fixed r.m.s. radius and $q \rightarrow \infty$, the brightness distribution (5) also tends to the Gaussian one.

Models (5) and (4) describe a class of compact sources with a fast brightness decrease. On the contrary, the power-law models $[6,7]$ describe a slow decrease at large $r$ :

$$
I_{\mathrm{PL}}(r)=\frac{p-1}{\pi R^{2}}\left[1+r^{2} / R^{2}\right]^{-p},
$$

where $p>1$ is the power index. Model (6) may be considered as an alternative to (5). The half-brightness radius of the source for this model is $R_{1 / 2}=R \sqrt{2^{1 /(p-1)}-1}$. Like (5), for the fixed r.m.s. radius and $p \rightarrow \infty$, the brightness distribution (6) tends to the Gaussian one. For small $p$ we have a "long-range" distribution. Throughout this paper we choose $p=3 / 2$ in order to have an asymptotic behavior analogous to the one of the accretion disc models (AD, see below).

The linear combinations of distributions (4), (5), (6) with diverse parameters yield rather a wide class of symmetric source models to fit any kind of data. On the other 
hand, (4) may be considered as a fiducial model to determine some parameters such as the source size and $(5,6)$ are useful in the case when in addition we are interested in the brightness behavior at large $r$.

The accretion disk (AD) of Shakura-Sunyaev [27] has a more complicated profile. This model gives the energy density of the radiation from the accretion disk around a non-rotating black hole as a function of radius. The (normalized) brightness distribution is

$$
I_{\mathrm{AD}}(r)=\frac{3 R \theta(r-R)}{2 \pi r^{3}}\left[1-\sqrt{\frac{R}{r}}\right]
$$

here $R$ being the radius of the inner edge of the accretion disk. For this $\mathrm{AD}$ model the half-brightness radius is $R_{1 / 2}=4 R$. This formula describes the total brightness integrated over all radiation frequencies. The maximum brightness of (7) is at $r=r_{m}=49 R / 36$. This model has the same behavior as the PL one (6) with $p=3 / 2$ for large $r$ while it is different for $r \sim R$.

For the blackbody radiation the disc temperature scales as $T \sim I_{\mathrm{AD}}^{1 / 4}$, whence the specific intensity is $\sim \lambda^{-5}\left(e^{h c / \lambda T}-1\right)^{-1}$ for the wavelength $\lambda[16]$ (AD1):

$$
I_{\mathrm{AD} 1}(r)=\frac{C_{\mathrm{AD} 1}}{R^{2}}\left[\exp \left(\frac{\kappa_{1} \rho^{3 / 4}}{\left(1-\rho^{-1 / 2}\right)^{1 / 4}}\right)-1\right]^{-1} \theta(r-R),
$$$$
\rho=r / R,
$$

where $\kappa_{1}=2.422$ and $C_{\mathrm{AD} 1} \approx 9.6$ is the normalization factor.

There is a simplified version (AD2) of the previous distribution [15] that can be defined as

$$
\begin{aligned}
& I_{\mathrm{AD} 2}(r)=\frac{C_{\mathrm{AD} 2}}{R^{2}}\left[\exp \left(\kappa_{2} \rho^{3 / 4}\right)-1\right]^{-1}, \\
& \rho=r / R \geq 1
\end{aligned}
$$

as distinct from [15] we introduced the constant $\kappa_{2}=$ 1.768 so as to have $R_{1 / 2} / R=2.021$ the same as in case of (8); in this case the $C_{\mathrm{AD} 2}=0.515$.

Though intensities (7), (8), (9) are quite different, their light curves in HAE may look rather similar. For the illustration we consider a linear caustic approximation (see, e.g., [28]) for the point source amplification

$$
K\left(y_{1}, y_{2}\right)=\theta\left(y_{2}\right) \sqrt{L / y_{2}}
$$

with the characteristic scaling parameter $L=1$. The corresponding convolutions of (7), (8), (9) and (4) with (10) are shown on Fig. 1. The main feature that distinguishes the accretion disk models from the Gaussian one is the concavity of the light curve owing to the dark "hole" in the accretion disk center. We remind, however, that the linear caustic approximation works well only in the case of the fold caustic crossing (far from the cusp) by a small source; therefore a detailed investigation is needed which must involve more complicated situations.

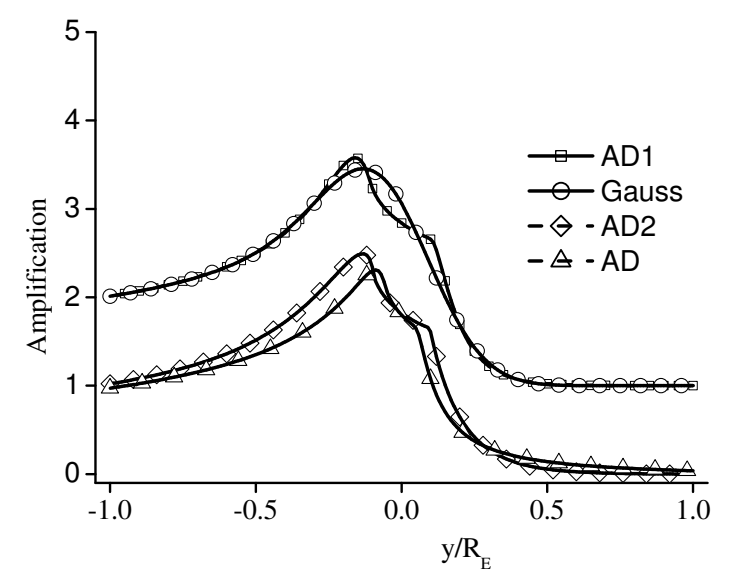

Fig. 1. Amplifications of the accretion disk models AD, $\mathrm{AD} 1, \mathrm{AD} 2$ and the Gaussian model (linear caustic approximation) as functions of distance $Y$ from the source center to the caustic. The "lightcurves" for AD1 and AD2 appear to be almost the same, so we shifted up the AD1 and Gaussian model curves by unity to avoid overlapping.

\section{SIMULATIONS}

\section{A. Initial parameters of the simulations}

To simulate the light curves we have used the "rayshooting" method with a direct calculation of each deflection angles. The parameters of numerical integration along with the parameters of the microlensing field are presented in Table 1 for microlensing by single microlenses and Table 4 for microlensing by binary microlenses. In calculations the Einstein ring radius was assumed $R_{\mathrm{E}}=1$ for all microlenses. The microlens positions were chosen in a random way with uniform distribution over the lens plane. The length of trajectory has been chosen long enough to have the caustic crossings events (far from the boundaries of the field), and the size of the microlensing field was chosen large enough to avoid boundary effects.

\begin{tabular}{|l|l|}
\hline \multicolumn{1}{|c|}{ Parameter } & \multicolumn{1}{|c|}{ Value } \\
\hline Resolution, pixels & $1.23 \times 10^{6}$ \\
Pixel size & $0.01 R_{\mathrm{E}}$ \\
Source trajectory length & $5 R_{\mathrm{E}}$ \\
Radius of field & $70 R_{\mathrm{E}}$ \\
Microlensing optical depth $(\sigma)$ & {$[0.1 ; 0.6]$} \\
\hline
\end{tabular}

Table 1. Parameters of simulation.

To compare the light curves for different source models we used the relative difference value

$$
\eta=2 \max _{t}\left(\frac{\left|K_{i}(t)-K_{j}(t)\right|}{K_{i}(t)+K_{j}(t)}\right)
$$

where $K_{i}(t)$ and $K_{j}(t)$ is an amplification for $i$-th and $j$ th model, respectively, along the trajectory of the source, which moves uniformly. 


\section{B. Light curves statistics}

Here we compare the light curves for Gaussian, PL, $\mathrm{LD}, \mathrm{AD}$ and AD2 source models with the brightness distributions from Section IIB. The simulations were performed for a set of 100 random realizations of the microlensing field with the microlensing optical depth $\sigma=0.3$ in order to have a possibility to compare with some of the results of the papers [29-32]. All the source models have the same half-brightness radius $R_{1 / 2}$, the light curves for all the source models have been calculated for the same microlensing fields. All the microlenses are static. The typical magnification pattern is shown on Fig. 2; here the speed of source is $V=1$.

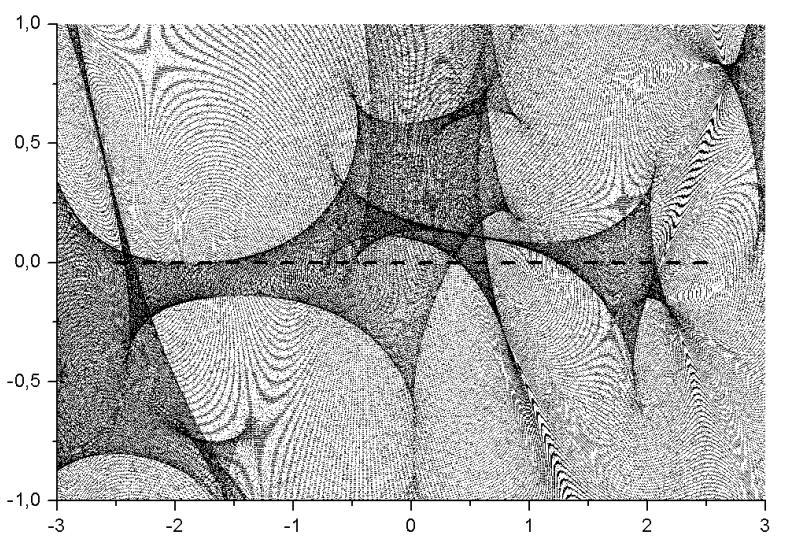

Fig. 2. The magnification pattern with the trajectory of the source in the source plane. The source moves from left to right along the straight line with uniform speed.

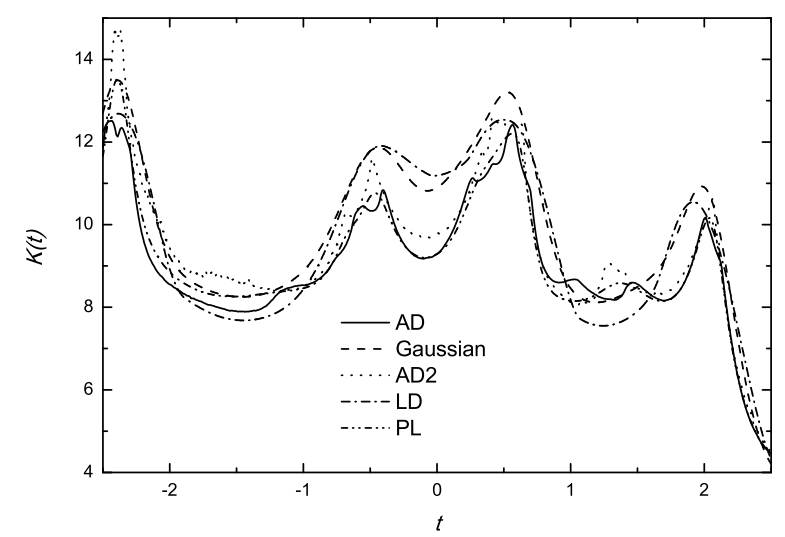

Fig. 3. "Light curves": amplification as a function of time for various source models that correspond to the magnification pattern of Fig. 2.

We present the results of simulations with the same half-brightness radius $R_{1 / 2}=0.21$; the power-law index was $p=3 / 2$ for the "long range" $\mathrm{PL}$ model; AD also corresponds to this class of the power-law asymptotic dependence. For the LD model we have chosen $q=1$.

From the "light curves" on Fig. 3 we observe a significant difference between the "compact" (LD and Gaussian) and the "long-range" models. The long-range character of the latter reveals itself even on considerable distances from the caustics, where we expect that the brightness of all the sources must have the same behavior as that of a point source. The differences between these two groups of models are essentially larger than the differences within each group (e. g. between Gaussian and LD models). This conclusion is confirmed by the results of the statistical considerations over 100 realizations shown in Table 2 for half-brightness radius $R_{1 / 2}=0.21$ as an example.

\begin{tabular}{|c|c|c|}
\hline$i$-th model & $j$-th model & $\eta$ \\
\hline AD & Gaussian & $0.074 \pm 0.0012$ \\
AD & AD2 & $0.085 \pm 0.002$ \\
AD & LD & $0.091 \pm 0.002$ \\
AD & PL & $0.038 \pm 0.001$ \\
Gaussian & AD2 & $0.073 \pm 0.0017$ \\
Gaussian & LD & $0.042 \pm 0.001$ \\
Gaussian & PL & $0.073 \pm 0.0013$ \\
AD2 & LD & $0.094 \pm 0.002$ \\
AD2 & PL & $0.052 \pm 0.0012$ \\
LD & PL & $0.090 \pm 0.002$ \\
\hline
\end{tabular}

Table 2. Relative difference between the light curves in HAE.

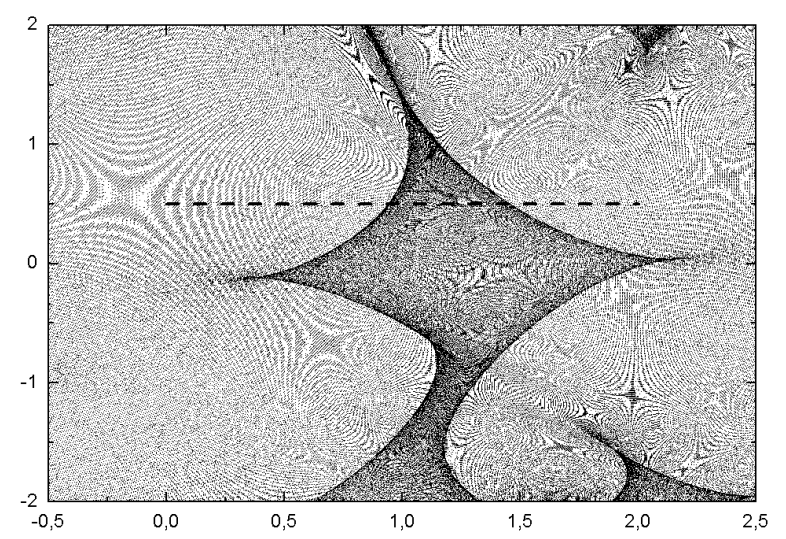

Fig. 4. Magnification pattern with simple caustic crossing events.

We note that the results of comparison may depend on the complexity of the caustics involved in our consideration. One can expect, for instance, that in the case of a complicated caustic crossing (such as the crossing of the fold caustics in the vicinity of cusp, intersection of dense aggregations of caustics, etc.) the light curve difference for various source models may be more significant. Our previous simulations involved all the possible realizations of the microlensing field that may involve such complicated caustic crossings. However, in the case of a concrete GLS we deal with a single light curve that may correspond to a simple caustic crossing which may be seen from the form of the light curve. Therefore, we may have a kind of an observational selection. Is it possible to apply the results of statistical simulations to a single light curve? So it is necessary to check whether this "complexity" affects the average value of $\eta$. In this connection we considered some modification of our statistical procedure: we have chosen such realizations rather simply 
("by eye") for the fold caustic crossings involved. However, the numerical results for simple caustic crossing events (e.g., magnification pattern for such an event is shown on Fig. 4), appeared to be nearly the same as that of Table 2. Though we must note that here the statistics have been worse because of a smaller number of simple caustic realizations, so this conclusion requires further verification.

\section{Gaussian fittings of the accretion disk and limb darkening models}

The above results concern a comparison of different models with the same $R_{1 / 2}$. However, in reality we do not know what radius should be used and one may ask why not to fit a light curve with different size parameters of different models. Therefore, we must check whether we can replace one model with a different one with some other source parameters to get better fitting.

\begin{tabular}{|c|c|c|}
\hline$i$-th model & $j$-th model & $\eta$ \\
\hline AD & Gaussian $\left(R_{1 / 2}=0.2\right)$ & $0.07 \pm 0.006$ \\
LD & Gaussian $\left(R_{1 / 2}=0.24\right)$ & $0.026 \pm 0.002$ \\
\hline
\end{tabular}

Table 3. Differences between models for fitted curves.

We have fitted the limb-darkening and accretion disk model light curves with the Gaussian models having different radii. The half-brightness radius of $\mathrm{LD}$ and $\mathrm{AD}$ models was $R_{1 / 2}=0.21 R_{\mathrm{E}}$ and that of the Gaussian source varied from $R_{1 / 2}=0.2 R_{\mathrm{E}}$ to $R_{1 / 2}=0.24 R_{\mathrm{E}}$; here $q=1$ for the LD model.

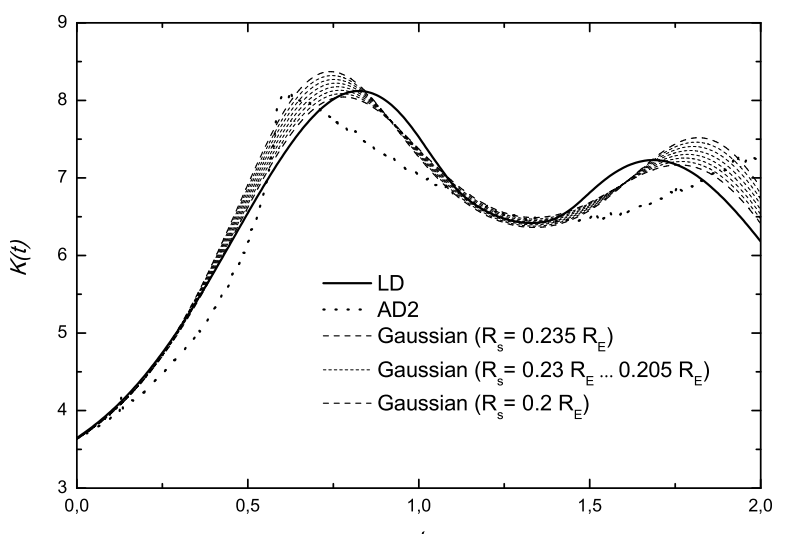

Fig. 5. Light curves of $\mathrm{LD}^{t}$ and AD1 models are fitted with the Gaussian source of different size that varies from $R_{1 / 2}=0.2 R_{\mathrm{E}}$ to $R_{1 / 2}=0.24 R_{\mathrm{E}}$ with the step $\delta R_{1 / 2}=0.001 R_{\mathrm{E}}$. These light curves correspond to the magnification pattern of Fig. 4.

As we found out (see Table 3 and Figure 5) the Gaussian source cannot reproduce all the models though for the class of the compact models the fitting results are rather good. For example, the LD model can be replaced by the Gaussian model with the other source size. However, this is a consequence of the similarity between light distributions (4) and (6).
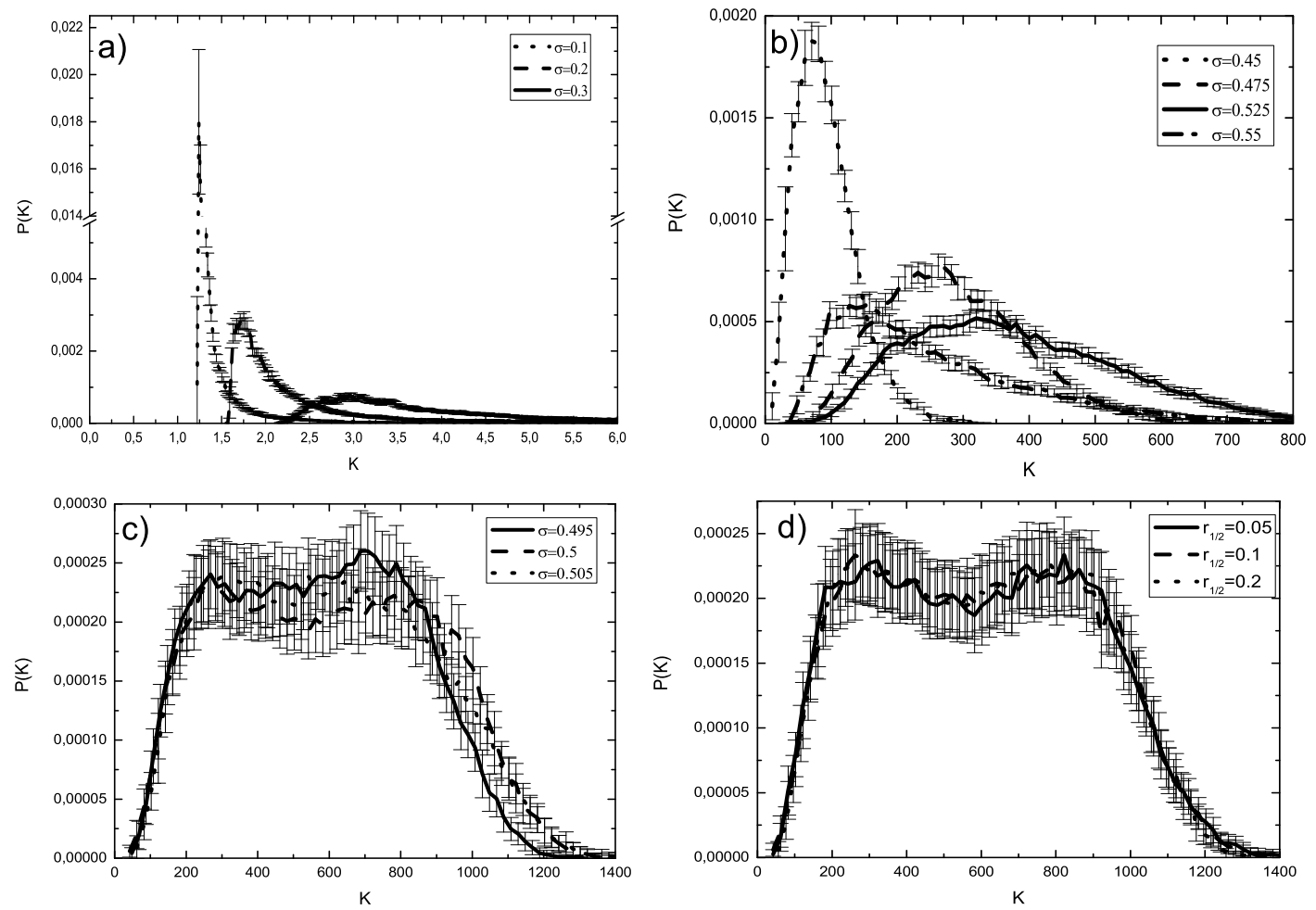

Fig. 6. Distribution of amplification coefficients for the Gaussian source $R_{1 / 2}=0.1$ for various optical depths: a) $\sigma=0.1 ; 0.2 ; 0.3$ and the step over $K$-axis is $\Delta K=0.02 ;$ b) $\sigma=0.45 ; 0.475 ; 0.525 ; 0.55$ and $\Delta K=10 ; \mathrm{c}) \sigma=0.495 ; 0.5 ; 0.505$ and $\Delta K=20$ and for different source radii for $\sigma=0.5$ and $\Delta K=20: \mathrm{d}) R_{1 / 2}=0.05 ; 0.1 ; 0.2$. 


\section{Optical depth effect on amplifications}

Microlensing variations of the source brightness is definitely affected by the properties of the microlenses in a deflecting galaxy: density, mass and position. In our calculations we have varied in a wide range the optical depth $\sigma$ to check the influence of the optical depth on the microlensing light curves. We have calculated over 500 realizations, each realization included microlenses with a random uniform distribution that we have built the histograms with amplification distributions for the Gaussian source over the trajectory in the case of different optical depths, the results are presented on Fig. 6 .

The distribution with two peaks that is obtained for $\sigma=0.5$ is much alike the distributions obtained in [33]. To test the reliability of the results we have used different random generators, changed the size of the field, the length of the trajectory and the source radius but the two-peak distribution arises any way when $\sigma=0.5$. It is also remarkable that the maximum of two peaks moves from smaller to bigger amplifications as $\sigma$ increases for the $\sigma$ close to $\approx 0.5$. This may be interpreted as follows. For small sigma the magnification pattern looks like many small structures with diamond-shaped caustics; the probability that the source intersects some of these structures is small. In this case a typical amplification will be near unity. As $\sigma$ increases, there are two types of regions: inside and outside caustics yielding two characteristic amplifications corresponding to two the maxima on Fig. 6. The values of these maxima roughly correspond to a relative part of the surface occupied by these regions. Further growth of $\sigma$ makes the picture more complicated but the bimodal structure is preserved at least up to $\sigma=0.6$.

\section{E. Impact of binary stars on microlensing}

Mao and Paczynski [34] pointed out that the light curve in the case of microlensing by a binary star will be quite different from the case of a single star. This will be in the case when the separation between companions of the binary system will be of the order of the Einstein radius and the impact parameter of the source trajectory will be of the same order. In the case of a strong Galactic microlensing, which is a rare event because of small microlensing optical depth, the observation of the corresponding light curve would give us a valuable information about this lensing binary system. However, in the light curves of extragalactic GLS with a huge caustic network it would be difficult to separate the binary star effects from other effects due to complicated caustics. In this connection we shall estimate a statistical relevance of the effect due to binary systems. We shall compare simulations of light curves in GLS with single stars to GLS with the analogous distribution of binaries. Binary systems were produced by splitting every initial point mass into two equal point masses separated by a distance in the range of $d \in[0.01 ; 0.05] R_{\mathrm{E}}$ that was selected randomly. The orientation in the binary system was also random. That is for every realization $(\mathrm{S})$ of single point masses ("stars" with the same mass $M$ ) we generate realization (B) of binaries with the mass $M / 2$ of components and with the same position of the centers as in the case of stars. All the masses were static. We calculated light curves (number of realizations of the microlens positions was about 100 for each case) for different microlensing optical depths, source half-brightness radii and different models of the source (Table 5).

\begin{tabular}{|l|c|}
\hline \multicolumn{1}{|c|}{ Parameter } & Value \\
\hline Resolution, pixels & $1.23 \times 10^{6}$ \\
Pixel size & $0.01 R_{\mathrm{E}}$ \\
Source trajectory length & $5 R_{\mathrm{E}}$ \\
Radius of field & $70 R_{\mathrm{E}}$ \\
Microlensing optical depth $(\sigma)$ & $0.3 ; 0.4 ; 0 ; 5$ \\
Number of microlenses & $1470 ; 1960 ; 2450$ \\
Distance in binary system $(d)$ & {$[0.01 ; 0.05] R_{\mathrm{E}}$} \\
\hline
\end{tabular}

Table 4. Parameters of simulation microlensing by binary-systems.

\begin{tabular}{|c|c|c|c|}
\hline model & $\sigma=0.3$ & $\sigma=0.4$ & $\sigma=0.5$ \\
\hline AD & $0.012 \pm 0.0008$ & $0.009 \pm 0,004$ & $0.05 \pm 0.0009$ \\
Gaussian & $0.009 \pm 0.0005$ & $0.006 \pm 0.0002$ & $0.004 \pm 0.0009$ \\
$\mathrm{PL}(\mathrm{p}=3 / 2)$ & $0.009 \pm 0.0005$ & $0.006 \pm 0.0003$ & $0.004 \pm 0.0009$ \\
$\mathrm{AD} 2$ & $0.03 \pm 0.0012$ & $0.02 \pm 0.0009$ & $0.006 \pm 0.0002$ \\
$\mathrm{LD}(\mathrm{q}=2)$ & $0.008 \pm 0.0005$ & $0.005 \pm 0.0002$ & $0.003 \pm 0.0009$ \\
\hline
\end{tabular}

Table 5. Relative difference $\eta$ between the light curves with and without binary microlenses $\left(R_{1 / 2}=0.2\right)$.

The difference in $\eta$-parameter in cases (B) and $(\mathrm{S})$ appears to be rather small. Nevertheless, the results show that, at least for some source models, it is possible to distinguish these cases by statistical analysis of the light curves at the modern level of photometric observations. The most significant difference between $(\mathrm{B})$ and $(\mathrm{S})$ is for the AD2 model and reaches up to $3 \%$ for $R_{1 / 2}=0.2$ and $\sigma=0.3$. For the AD2 (9) model and $R_{1 / 2}=0.15, \sigma=0.3$ the difference is $4 \%$ and for $R_{1 / 2}=0.1$ it amounts to $7 \%$. The reason for this is because the smaller source "probes" the inner structure of the deflecting galaxy more precisely.

\section{DISCUSSION}

Before we formulate the main results we must make some reservations and caveats.

Firstly, it is impossible to get a complete information about the brightness distribution from the light curve observations only. The information from separate HAE only, without making recourse to the whole light curve, etc., is still more limited. The observations provide only a one-dimensional luminosity profile of the source (integrated along the caustic); then without using some additional information we cannot determine even the source size because we do not know the value and direction of the source velocity, ellipticity and orientation with respect to the caustic. 
Secondly, even under the assumption of a circularly symmetric source and known normal velocity with respect to the fold caustic, the determination of the luminosity profile ("de-projection") is a kind of ill-posed mathematical problems: small variations of input data may lead to a considerable changes of the solution. A standard way to relax this difficulty involves additional restrictions and/or using some simple "fiducial" models for brightness distribution. Some of these models have been considered in this paper (Gaussian, power law and accretion disc). However, it should be clearly understood that the real picture of the central quasar region is more complicated than our simple brightness distributions; these models can be considered rather as some reference models. On the other hand, in view of the present-day accuracy of observations, it is somtimes difficult to distinguish even these simple source models on the basis of observational data. For example, the authors of [16] argue that the accretion disk can be modelled with any brightness profile (Gaussian, uniform, etc.), and this model will agree with the available data provided that an appropriate source size is chosen. On the other hand, a number of authors [5-7,12-17] discussed delicate questions concerning the determination of a fine quasar structure from HAE. For example, the authors of [14] wrote that the GLITP data [2] on Q2237+0305 admit only accretion disc models (see also $[12,13]$ ). Obviously, the presence of an accretion disk in a central region of quasar is beyond any doubts as well as the fact that the real appearance of the quasar core can be quite different from the simplified models. What we can really do (and this is one of the problems discussed in this paper) is to compare the results of fitting with concrete reference models [12, 13, 15-17, 35-37].

In this paper we performed statistical simulations of microlensing processes with different reference source models: Gaussian and power-law model and some accretion disc models. We considered two types of mass distributions single stars (S) and binaries (B) with uniform static disposition in space. The masses of stars were equal. The results can be considered as moderately optimistic. We have shown that one can choose between some reference models of the source in GLS which lead to different values light curves. This difference is characterized by $\eta$-parameter which sometimes amounts the
$9 \%$. The difference between mass distributions $(\mathrm{S})$ and (B) is less noticeable.

Though we deal with complete light curves, one must remember that the source structure manifests itself only in the HAEs; far from the caustics the source looks like the point one and all the information about its structure is being lost. Clearly, our results concerning $\eta$ in fact deal with HAE just where we can obtain the maximum difference of amplifications for different source models. Also, our results are obtained from a number of different realizations of the microlensing mass distribution, but in observations we have only one light curve which is obtained for a very limited interval. In reality we have no statistical ensemble, so statistical simulations provide us with some average qualitative estimates; they may differ from the real values for a concrete GLS. This raises the cost of individual HAE observations and their treatment.

The authors would like to thank V. N. Dudinov for helpful suggestions and discussions. The main results of Section III were presented at the Sixth Scientific Conference in Honor of Bohdan Babiy "Selected Issues of Astronomy and Astrophysics" , October 4-6, 2011, Lviv. This work has been supported in part by "Cosmomicrophysics" program of National Academy of Sciences of Ukraine. V. M. S. and V. I. Z acknowledge the partial support of Swiss National Science Foundation within SCOPES project IZ73Z0_128040.

\section{Appendix 1}

The size of an object can be introduced by means of the coherence area, which is known from statistical optics [25]. As distinct from [25], this can be rewritten in terms of the brightness distribution $I(\mathbf{r})$ without recourse to the spatial Fourier transform. We have then a definition of a characteristic source area as follows

$$
S=\frac{\left(\int I(\mathbf{r}) d^{2} \mathbf{r}\right)^{2}}{\int I^{2}(\mathbf{r}) d^{2} \mathbf{r}}
$$

Then we can define a characteristic radius $R_{c}$ of the source (at least in a circular symmetric case) by putting

$$
S=\pi R_{c}^{2}
$$

For example, in case of the Gaussian source (4) we have $R_{c}=\sqrt{2} R$.
[1] B. Grieger, R. Kayser, S. Refsdal, Astron. Astrophys. 194, 54 (1988).

[2] D. Alcalde et al., Astrophys. J. 572, 729 (2002).

[3] A. Udalski et al., Acta Astron. 56, 293 (2006).

[4] P. R. Woźniak et al., Astrophys. J. 529, 88 (2000).

[5] M. B. Bogdanov, A. M. Cherepashchuk, Astron. Rep. 46, 626 (2002).

[6] V. N. Shalyapin, Astron. Lett. 27, 150 (2001).

[7] V. N. Shalyapin et al., Astrophys. J. 579, 127 (2002).

[8] J. S. Wyithe, R. L. Webster, E. L. Turner, Mon. Not. Roy. Astron. Soc. 309, 261 (1999).

[9] J. S. Wyithe, R. . Webster, E. L. Turner, Mon. Not. Roy.
Astron. Soc. 318,762 (2000).

[10] J. S. Wyithe, R. L. Webster, E. L. Turner, D. J. Mortlock, Mon. Not. Roy. Astron. Soc. 315, 62 (2000).

[11] A. Yonehara, Astrophys. J. 548, L127 (2001).

[12] T. Anguita et al., Astron. Astrophys. 480, 327 (2008).

[13] R. Gil-Merino, J. Gonzalez-Cadelo, L. J. Goicoechea, V. N. Shalyapin, G. F. Lewis, Mon. Not. Roy. Astron. Soc. 371, 1478 (2006).

[14] L. J. Goicoechea, D. Alcalde, E. Mediavilla, J. A. Muñoz, Astron. Astrophys. 397, 517 (2003).

[15] C. S. Kochanek, Astrophys. J. 605, 58 (2004).

[16] M. J. Mortonson, P. L. Schechter, J. Wambsganss, As- 
trophys. J. 628, 594 (2005).

[17] V. G. Vakulik, R. E. Schild, G. V. Smirnov, V. N. Dudinov, V. S. Tsvetkova, Mon. Not. Roy. Astron. Soc. 382, 819 (2007).

[18] A. N. Alexandrov, V. I. Zhdanov, Mon. Not. Roy. Astron. Soc. 417, 541 (2011).

[19] A. N Alexandrov, V. I Zhdanov, E. V. Fedorova, Astron. Lett. 36, 329 (2010).

[20] A. N Alexandrov, V. M. Sliusar, V. I Zhdanov, Ukr. J. Phys. 56, 389 (2011).

[21] R. Kayser, S. Refsdal, R. Stabell, Astron. Astrophys. 406, 24 (1986).

[22] P. Schneider, A. Weiss, MPA Rep. 311, 46 (1987).

[23] J. Wambsganss, B. Paczynski, P. Schneider, Astrophys. J. 358, 33 (1990).

[24] I. Hubeny, V. Hubeny, Astrophys. J. 505, 558 (1998).

[25] J. W. Goodman, Statistical Optics (New-Yokr, J. Wiley and Sons, 1985).

[26] M. Dominik, Mon. Not. Roy. Astron. Soc. 353, 69 (2004).

[27] N. I. Shakura, R. A. Sunyaev, Astron. Astrophys. 24,
337 (1973).

[28] P. Schneider, J. Ehlers, E. E. Falko, Gravitational Lenses (New York, Springer, 1992).

[29] X. Dai, E. Agol, M. W. Bautz, G. P. Garmire, Astrophys. J. 589, 100 (2003).

[30] E. V. Fedorova, V. I. Zhdanov, C. Vignali, G. G. C. Palumbo, Astron. Astrophys. 490, 989 (2008).

[31] R. Schmidt, R. L. Webster, F. G. Lewis, Mon. Not. Roy. Astron. Soc. 295, 488 (1998).

[32] V. Vakulik et al., Astron. Astrophys. 447, 905 (2006).

[33] V. S. Tsvetkova, V. M. Shulga, V. G. Vakulik, G. V. Smirnov, V. N. Dudinov, A. A. Minakov, Kin. Phys. Cel. Bod. 25, 282009.

[34] S. Mao, B. Paczynski, Astrophys. J. 374, 37 (1991).

[35] S. Poindexter, C. S. Kochanek, Astrophys. J. 712, 658 (2010).

[36] S. Poindexter, C. S. Kochanek, Astrophys. J. 712, 668 (2010).

[37] S. Poindexter, N. Morgan, C. S. Kochanek, Astrophys. J. 673, 34 (2008).

\title{
СИМУЛЯЦІЇ ГРАВІТАЦІЙНОГО МІКРОЛІНЗУВАННЯ: МОДЕЛІ ПРОТЯЖНОГО ДЖЕРЕЛА ТА ВПЛИВ ПОДВІЙНИХ ЗІР
}

\author{
В. М. Слюсар, В. І. Жданов, О. М. Александров \\ Астрономічна обсерваторія Київсъкого начіонального університету імені Тараса Шевченка
}

\begin{abstract}
У позагалактичній гравітаційно-лінзовій системі через ближчу галактику спостерігають декілька зображень віддаленого квазара. Спостереження кривих блиску різних зображень квазара дає важливу інформацію про центральну область квазара. Ми проводимо статистичні симуляції кривих блиску мікролінзованих зображень для визначення можливості виявити моделі джерела з різними розподілами яскравості. Розглянуто випадки розподілу мас у лінзуючій галактиці: (i) випадковий розподіл одиничних зір і (ii) аналогічний розподіл із подвійними зорями. Ми встановили, що різниця між кривими блиску для гауссівського розподілу яскравості й моделі акреційного диска з однаковими розмірами диска може в деяких випадках досягати 8\%. Якщо підганяти моделі акреційного диска й моделі "limb-darkening" гауссівським розподілом поверхневої яскравості з довільному розмірі джерела, то різниця між моделями може бути зменшена до $7 \%$ і $2.6 \%$ відповідно. Порівняння результатів показують, що різниці між кривими блиску для випадків (i) i (ii) $€$ на межі статистичної похибки, але деякі криві блиску можуть суттєво відрізнятися.
\end{abstract}

